\title{
GPS low noise amplifier with high immunity to wireless jamming signals and power control option
}

\author{
H. Schulz ${ }^{1}$, H.-J. Golberg ${ }^{1}$, F. Gruson ${ }^{2}$, M. Durler ${ }^{3}$, and S. J. Spiegel ${ }^{3}$ \\ ${ }^{1}$ Atmel Germany GmbH, Theresienstrasse 2, D-74072 Heilbronn, Germany \\ ${ }^{2}$ Department of Electron Devices and Circuits, University of Ulm, D-89081 Ulm, Germany \\ ${ }^{3}$ U-Blox AG, Zurcherstrasse 68, Thalwil, CH-8800, Switzerland
}

\begin{abstract}
A SiGe GPS low noise amplifier with power control option and high immunity to wireless jamming signals is presented. These novel features applied to Atmel's ATR0610 GPS LNA allow significant power saving at the radio interface while meeting the out-of-band linearity requirements. The results show the noise figure less than $2.1 \mathrm{~dB}$, including the embedded pre-select filter, and out-of-band IIP3 above $+8 \mathrm{dBm}$ in the frequency range between $1.8 \mathrm{GHz}$ and $2 \mathrm{GHz}$ with $3 \mathrm{~mA}$ current consumption. The GPS system performance shows GPS sensitivity below $-141 \mathrm{dBm}$ with $5 \mathrm{~ms}$ integration interval.
\end{abstract}

\section{Introduction}

The Atmel ATR0610 GPS LNA shows an optimized tradeoff between low noise, high gain, low power consumption, stability, linearity and very small $2.0 \mathrm{~mm} * 1.6 \mathrm{~mm}$ PLLP-6L package. The implemented power control option is an additional feature, which allows significant power saving in the GPS system. The integrated output matching network and the pre-matched input of this LNA minimizes the number of external components. In order to increase the immunity to high jamming signals an external pre-select filter was designed.

\section{LNA design}

Figure 1 shows the schematic diagram of the ATR0610 GPS LNA. The cascode input stage reduces the capacitive loading associated with the Miller effect. Simultaneous input power and noise matching requires that the condition $\Gamma_{o p t}=\mathrm{S}_{11}^{*}$ is valid, where $\Gamma_{o p t}$ is the reflection coefficient for minimum noise figure. To match the optimum noise matching condition with minimum number of external components, the di-

Correspondence to: $\mathrm{H}$. Schulz

(holger.schulz@hno.atmel.com) mensions of the transistor $\mathrm{Q}_{1}$ in the presence of inductive emitter series degeneration, were optimized [1].

To reduce the noise figure, the inductive series feedback, $\mathrm{L}_{e}$ in Fig. 1, was realized with a bond wire and the package lead. The remaining imaginary part of $\Gamma_{o p t}$ and $S_{11}^{*}$ is almost completely compensated by the use of a series inductor, $\mathrm{L}_{i n}$, realized with the bond wire and the package lead at the base of $\mathrm{Q}_{1}$. Since the large common emitter device, $\mathrm{Q}_{1}$, dominates the noise figure, a 2-finger common base HBT, $\mathrm{Q}_{2}$, can be used at the output stage $[2,3]$. The base of this device is de-coupled with the MIM capacitor, $\mathrm{C}_{b}$.

The bias network, PTAT bias cell of Fig. 1, provides:

- Stable bias current versus process variations.

- Compensation to the inevitable drop of the transconductance $\mathrm{g}_{m}$ of the bipolar transistor with temperature.

- Power down pin with CMOS-compatible input range.

- Bias current proportional-to-absolute-temperature (PTAT).

Figures $2 \mathrm{a}$ and $2 \mathrm{~b}$ show two applications of the ATR0610 GPS LNA. The LNA for GPS standalone applications, Fig. 2a, requires only an L-type input matching network using standard SMD components to achieve the power matching. Due to the optimized HBT, $\Gamma_{\text {opt }} \approx \mathrm{S}_{11}^{*}$, an excellent noise figure result is obtained as shown in Fig. 3. To improve the jamming immunity to wireless communication signals, the input network of Fig. 2a was modified, resulting in the topology of Fig. $2 \mathrm{~b}$. The shunt resonator, $\mathrm{C}_{3}$ and $\mathrm{L}_{3}$, causes a notch in the transfer function at $1.8 \mathrm{GHz}$, giving at least $5 \mathrm{~dB}$ additional attenuation at the lowest frequency of the DCS transmission band. The series resonator, $\mathrm{C}_{2}$ and $\mathrm{L}_{2}$, introduces a second notch around $3 \mathrm{GHz}$. It significantly attenuates the interference due to the PCS band signals [4] and improves the noise and power matching to the LNA input. The blocking capacitor between the antenna and the LNA, $\mathrm{C}_{\text {block }}$, attenuates the signals in the $900 \mathrm{MHz}$ band by at least 

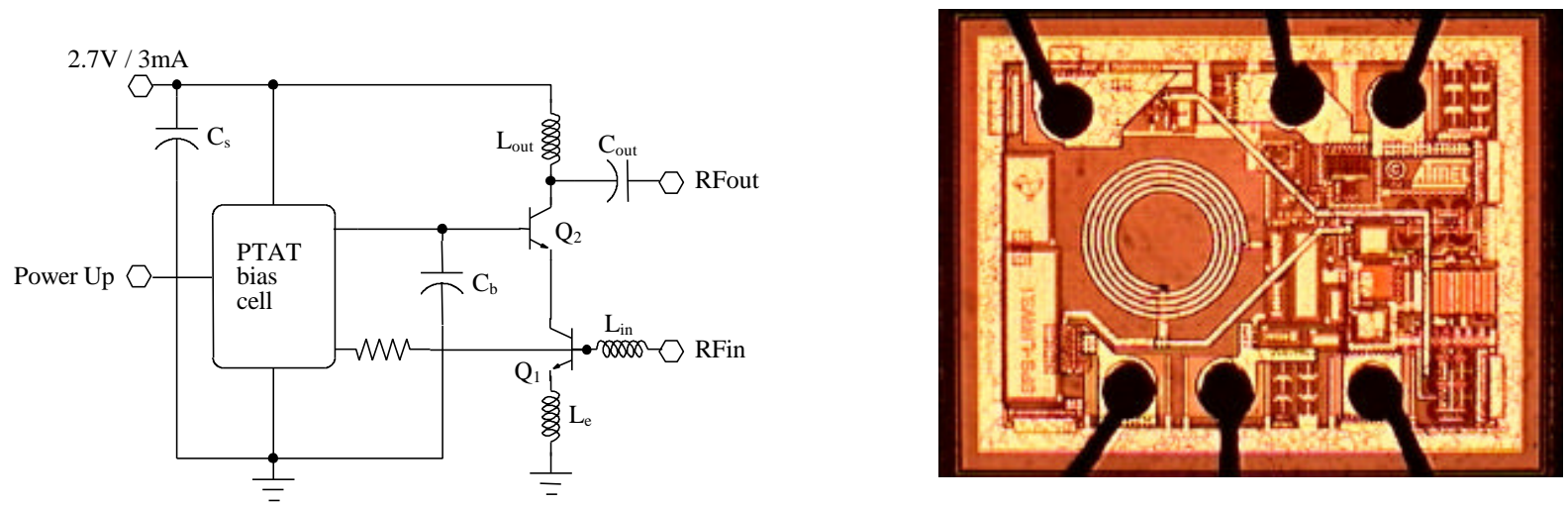

Fig. 1. Schematic and microphotograph of ATR0610 GPS LNA with power control option.

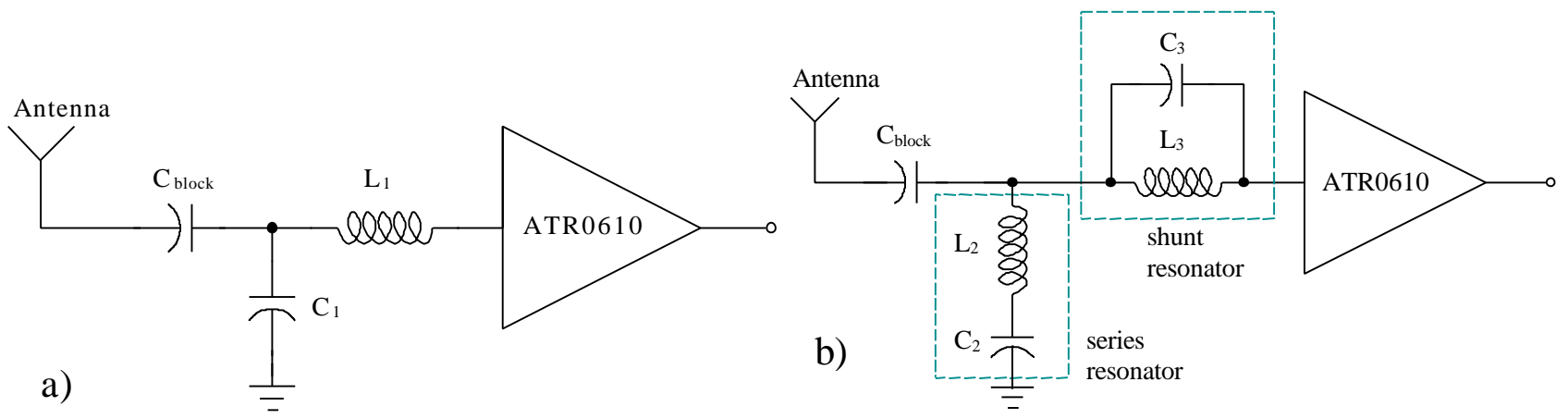

Fig. 2. Input application circuitry of ATR0610 GPS LNA (a) L-type network, (b) 2nd order input network.

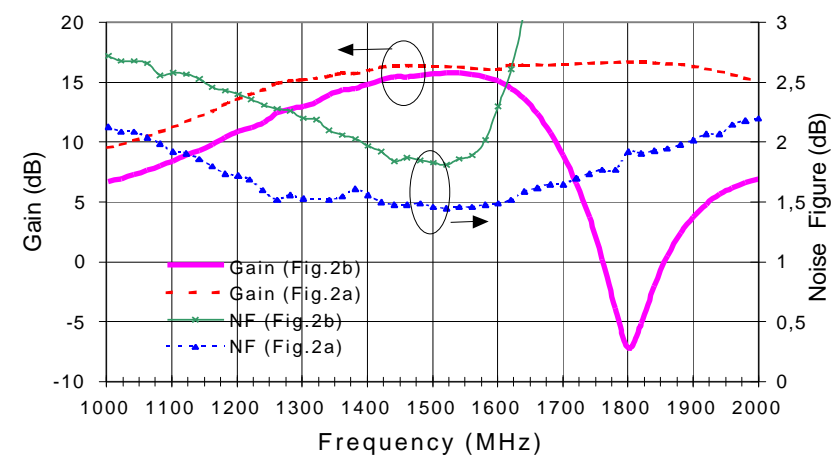

Fig. 3. Measured gain and noise figure of the ATR0610 GPS LNA.

$5 \mathrm{~dB}$. Owing to the small frequency gap between the GPS band $(1575 \mathrm{MHz})$ and the DCS transmission band, the most critical component is the shunt resonator, which needs to provide sufficient attenuation at $1710 \mathrm{MHz}$ with minimum noise degradation at GPS frequency. The out-of-band attenuation with less than $0.6 \mathrm{~dB}$ degradation on the noise figure was obtained with the modified input network, as indicated by the gain curves in Fig. 3. Finally, the noise figure degradation at GPS frequency in the presence of $\mathrm{CW}$ blocking at $1.75 \mathrm{GHz}$ and $1.95 \mathrm{GHz}$ is presented in Fig. 4.

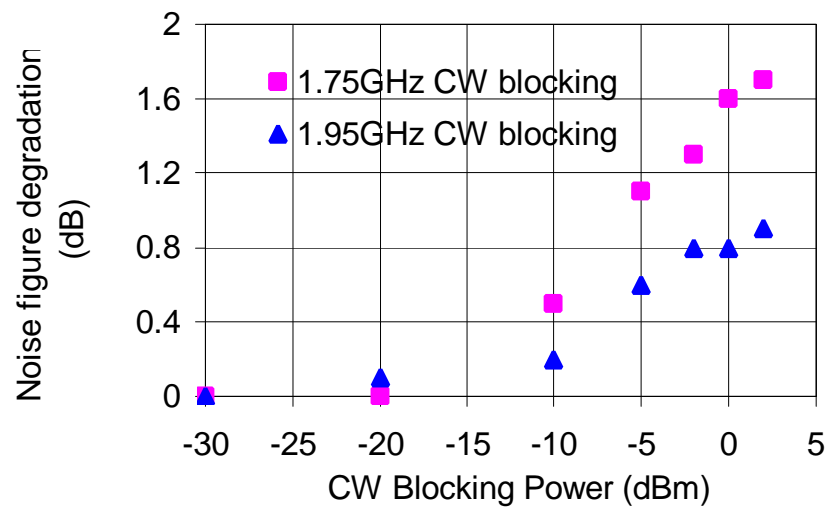

Fig. 4. Noise figure degradation in presence of CW jamming signals.

\section{System integration}

Integration of the ATR0610 GPS LNA is shown in Fig. 5. The power control of the low noise amplifier and the RF processor [5] of the entire radio receiver are supplied through a common control input. Substantial power saving is obtained in the radio interface by switching off the high frequency stages, except the reference clock generator. To the authors' knowledge, the ATR0610 LNA is the only standalone GPS LNA with power control option. The benefits of the standalone GPS LNA with power control option are observed in 


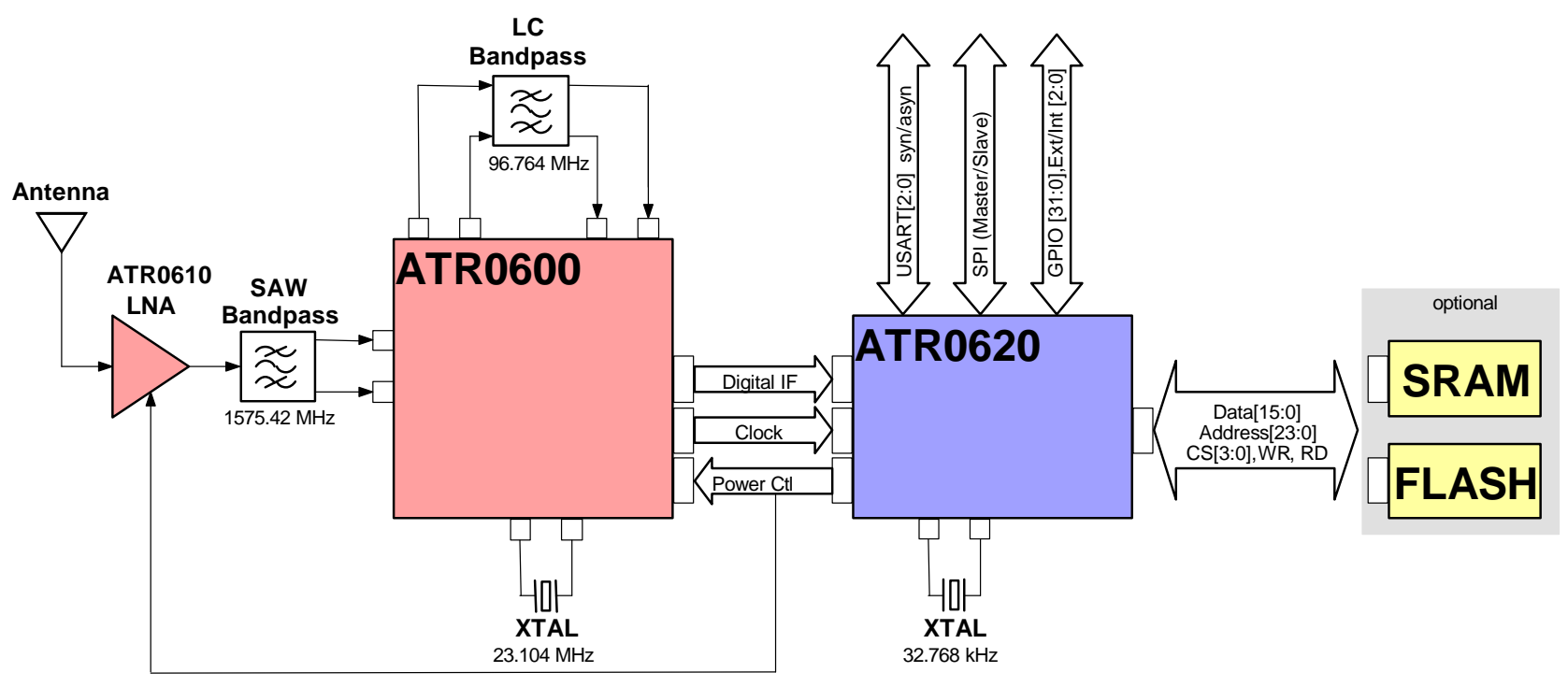

Fig. 5. Block diagram of the GPS receiver indicating the integration of the ATR0610 GPS LNA.

the portable mobile communication and applications using an external active GPS antenna. For portable mobile communication, the standalone GPS LNA provides an increase of the intra-system isolation between the GPS antenna and the distinct regions of the circuitry [6, 7]. High sensitivity, $5 \mathrm{~ms}$ integration time, and high immunity to jamming signals in the frequency range occupied by wireless communication systems were simultaneously obtained, as outlined in Table 1. For active GPS antenna applications, i.e. GPS antennas with an LNA, the novel power control feature in the ATR0610 GPS LNA offers the flexibility for power saving at the radio interface.

\section{Conclusions}

A GPS low noise amplifier based on Atmel's SiGe1 technology has been developed. The GPS LNA for GPS standalone and GPS integrated into wireless communication systems showed $1.6 \mathrm{~dB}$ and $2.0 \mathrm{~dB}$ noise figure respectively with $3 \mathrm{~mA}$ current consumption. The novel integration of features in the ATR0610 GPS LNA, the power control option and the jamming immunity characteristics, allowed the optimization of the power consumption of the complete GPS receiver without compromising the linearity requirements of the system.

\section{References}

[1] Götzfried, R., Beisswanger, F., Gerlach, S., Schüppen, A., Dietrich, H., Seiler, U., Bach, K. H., and Albers, J.: "RFIC's for Mobile Communication Systems Using SiGe Bipolar Technology", 1998 IEEE Transactions on Microwave Theory and Techniques, May 1998.

[2] Schumacher, H., Erben, U., and Gruhle, A.: "Low Noise Performance of SiGe Heterojunction Bipolar Transistor", 1994 IEEE
Table 1. Summary of the ATR0610 GPS LNA and the GPS system results.

\begin{tabular}{lll}
\hline Parameter & ATR0610 LNA & GPS system \\
\hline CNR degradation & & \\
\hline L-type input network & $1.5 \mathrm{~dB}$ & - \\
\hline 2nd order input network & $<2.1 \mathrm{~dB}$ & $4.9 \mathrm{~dB}$ \\
\hline $3 \mathrm{~dB}$ SNR degradation by CW blocking & $>-2 \mathrm{dBm}$ & $>-4 \mathrm{dBm}$ \\
CW blocking at $1.7 \mathrm{GHz}$ & $>+2 \mathrm{dBm}$ & $>+2 \mathrm{dBm}$ \\
CW blocking at $1.75 \mathrm{GHz}$ & $>0 \mathrm{dBm}$ & $>0 \mathrm{dBm}$ \\
CW blocking at $1.8 \mathrm{GHz}$ & $>+4 \mathrm{dBm}$ & $>+2 \mathrm{dBm}$ \\
CW blocking at $1.95 \mathrm{GHz}$ & & \\
\hline
\end{tabular}

IEEE Microwave and Millimeter-Wave Monolithic Circuit Symposium, 1994.

[3] Erben, U., Schumacher, H., Schüppen, A., Dietrich, H., and Arndt, J.: "Application of a Production Ready SiGe HBT Process to 1.9, 5.7 and 10 GHz Low Noise MMIC's", 1998 IEEE Topical Meeting on Silicon Monolithic Integrated Circuits in RF Systems, September 1998.

[4] Spiegel, S. J.: "GPS-RF system requirements for handset applications”, 2002 IEEE MTT-S Int. Microwave Symp. Workshop., June 2002.

[5] Golberg, H. J.: "RF design and implementation issues of GPS front-ends", 2002 IEEE MTT-S Int. Microwave Symp. Workshop., June 2002.

[6] Spiegel, S. J., Thiel, A., Nussbaumer, S., and Kovacs, I.: "Characterization of GPS receivers for mobile systems", 2002 Proceedings of the Institute of Navigation, September 2002.

[7] Spiegel, S. J., Thiel, A., Nussbaumer, S., Kovacs, I., and Durler, M.: "Improving the isolation of GPS receivers for integration with wireless communication systems", Accepted to publication at RFIC symposium digest, June 2003. 